\title{
SITIOS COSTARRICENSES DE NOTICIAS EN FACEBOOK: ¿QUÉ “LIKEAN”, COMENTAN Y COMPARTEN SUS USUARIOS?
}

\section{COSTA RICAN NEWS OUTLETS ON FACEBOOK: WHAT DO USERS LIKE, COMMENT AND SHARE?}

\author{
Ignacio Siles González* \\ Pedro Campos Acuña** \\ Andrés Segura Castillo***
}

\section{RESUMEN}

En este artículo, se discute cómo usuarias y usuarios interactúan con contenido noticioso publicado por sitios costarricenses de noticias en Facebook. Utilizando una metodología mixta que combinó el análisis de redes sociales (social network analysis) y el análisis de contenido, se examinó la información publicada por seis sitios costarricenses de noticias durante cuatro meses de 2016. Se identificó una clara preferencia de los usuarios por noticias sobre asuntos no públicos, como sucesos, fútbol y entretenimiento. En cambio, las noticias sobre asuntos públicos, como política, economía y gobierno, recibieron relativamente poca interacción de parte de las usuarias y los usuarios. Asimismo, se analizó una diferencia estructural en cómo las personas interactuaron con la información publicada por varios sitios informativos. Se concluye con una discusión de las oportunidades analíticas que ofrece el estudio de la circulación de noticias en Facebook.

PALABRAS CLAVE: ANÁLISIS * REDES SOCIALES * FLUJO DE NOTICIAS * MEDIOS DE
COMUNICACIÓN * INTERNET

ABSTRACT

This paper discusses how users interact with news content published by Costa Rican online news sites on Facebook. Drawing on a mixed-method research design that combined social network analysis and content analysis, we examined the data published by six major Costa Rican news sites during four months of 2016. We identified a clear user preference for content about non-public affair issues, such as crimes, sports, and entertainment. In

* Escuela de Ciencias de la Comunicación Colectiva, Universidad de Costa Rica (UCR). ignacio.siles@ucr.ac.cr

** Escuela de Ciencias de la Comunicación Colectiva, Universidad de Costa Rica (UCR). dg.pcampos@gmail.com

*** Laboratorio de Investigación e Innovación Tecnológica, Universidad Nacional Estatal a Distancia (UNED). asegurac@uned.ac.cr 
contrast, news about public affairs, such as politics, economy, and government, received relatively much less interaction. We also examined a structural difference in how users interacted with the content published by news sites. We conclude with a discussion of the analytical opportunities afforded by the circulation of news on Facebook.

KEYWORDS: ANALYSIS * SOCIAL MEDIA * NEWS FLOW * MEDIA * INTERNET

\section{INTRODUCCIÓN}

El consumo de noticias a nivel mundial ha migrado paulatinamente a Internet. Según el Pew Research Center:

entre millenials ${ }^{1}$, Facebook es por mucho y de lejos la fuente más común de noticias sobre el gobierno y la política. [...] [A]proximadamente 6 de cada 10 millenials $(61 \%)$ que utilizan la Web reportaron haber obtenido noticias políticas en Facebook. Esto es 17 puntos porcentuales más que la siguiente fuente de noticias más consumida por millenials (cnN con 44\%) (2015, párr. 1, cursiva del autor).

Este tipo de investigaciones sugiere que el acceso a diversas tecnologías, tales como dispositivos móviles (teléfonos celulares y tablets), ha facilitado las condiciones de lectura de contenidos noticiosos (Meijer y Kormelink, 2015).

De manera similar, el estudio anual realizado por unimer para El Financiero señala que el consumo de noticias en redes sociales ha ganado un auge notable en Costa Rica desde hace algunos años. En ese informe, se detalla que, para el 2015 , un $40 \%$ de la población consultada en el país reportaba el consumo de noticias como una de las cuatro principales actividades realizadas en Facebook (unimer, 2015). Este crecimiento de las noticias online ha acompañado

1 Aunque las definiciones varían, el término millenial suele referirse a "adolescentes y personas en sus veintes que están haciendo el pasaje a la edad adulta a inicios del nuevo milenio" (Pew Research Center, 2010, párr. 1). de manera paralela una disminución considerable del consumo de noticias en los llamados medios de comunicación "tradicionales" (la televisión, la radio y los medios impresos). Por ejemplo, una encuesta realizada por el Centro de Investigación en Estudios Políticos (CIEP) de la Universidad de Costa Rica para el Semanario Universidad notó que Internet constituye la única fuente de noticias en crecimiento en Costa Rica. Por el contrario, todos los demás medios de comunicación perdieron audiencia en el país (aunque la televisión se mantiene como el principal medio para consumir noticias) (Araya, 2016).

Este artículo contribuye a una mejor comprensión de los comportamientos que tienen las personas usuarias de perfiles de sitios costarricenses de noticias en Facebook. Se reportan algunos resultados de un estudio sobre patrones de interacción con noticias en Facebook en el país durante 2016. A diferencia de otras investigaciones, este estudio se centra en el comportamiento de las personas usuarias según lo registrado por Facebook y no en lo que estas personas dicen haber leído o consumido en esta plataforma. Como afirma Bird (2014), "las personas tienen la tendencia a aumentar la cantidad de noticias que leen cuando dan un reporte. La observación puede contradecir los reportes de atención a noticias sobre asuntos públicos que muchas personas se sienten obligadas a brindar" (p. 494), (se brinda una definición de qué se entiende por asuntos públicos y asuntos no públicos en la siguiente sección). Esta tendencia ha limitado la investigación sobre consumo de noticias (Madianou, 2010). Con el propósito de superar ese sesgo, se realizó un análisis de contenido a un conjunto 
de noticias y se examina qué tipo de contenidos obtuvo más interacciones de parte de usuarios, mediante prácticas como dar like o "me gusta", compartir contenidos o comentar las noticias en Facebook. Al realizar este ejercicio, se observó una clara preferencia de las usuarias y los usuarios por temas como sucesos, deportes $y$ entretenimiento.

Asimismo, se evidenciaron comportamientos estructurales de quienes leen en Facebook. Por estructurales, se entienden los patrones $y$ tendencias subyacentes que caracterizan los comportamientos de personas cuando interactúan con los contenidos publicados por sitios de noticias en esta red social a lo largo del tiempo. Para identificar estos patrones, se empleó el análisis de redes sociales (social network analysis $)^{2}$. De ese modo, se demostraron diferencias sustantivas en los comportamientos de las personas que interactúan con sitios de noticias en Facebook. Por ejemplo, se concluyó que mientras los usuarios de perfiles como CRHoy y Diario Extra tienden a leer un número considerable de noticias aunque no estén vinculadas temáticamente, aquellos de perfiles como $L a$ Nación tienen un comportamiento más fragmentado de interacción con las noticias, es decir, hacen clic en un contenido pero no en los otros que se les ofrece.

Sobre la base del conjunto de resultados analizados, se concluye con una discusión sobre algunas implicaciones del estudio, la complejidad del fenómeno de interés y algunas líneas de investigación que surgen al emplear este tipo de aproximaciones teórico-metodológicas para estudiar la circulación de contenido noticioso en Facebook.

\section{APROXIMACIONES TEÓRICAS: LA "BRECHA DE} LAS NOTICIAS” VISTA DESDE EL DATA SCIENCE

Esta investigación está fundamentada en una articulación interdisciplinaria de conocimientos teóricos provenientes de dos campos específicos: estudios en Comunicación y

$2 \quad$ Esta metodología también es conocida como "análisis estructural" o "análisis estructural de redes” (Wellman, 1997). principios de Data Science (ciencia y analítica de los datos).

Los estudios en Comunicación permiten comprender mejor las prácticas y motivaciones de quienes usan sitios de noticias online al interactuar con contenido noticioso. Asimismo, este campo de conocimientos ayuda a identificar los tipos de noticias que consumen usuarios y sus implicaciones para la vida democrática de una sociedad. Algunas investigaciones han sugerido que las personas tienen una clara preferencia por noticias sobre asuntos no públicos, es decir, aquellos temas que no necesariamente contribuyen a la formación de la esfera pública en su sentido más tradicional (es decir, burguesa según Habermas, 1989). Dentro de este tipo de contenidos destacan, por ejemplo, informaciones sobre sucesos, crímenes, deportes, entretenimiento, clima, etc. En cambio, las noticias sobre asuntos públicos tienen que ver con política, gobierno, funcionarios públicos (notablemente el presidente de la república e inclusive candidatos a ocupar cargos públicos), el acontecer del país, economía y asuntos internacionales, entre otros.

Boczkowski y Mitchelstein (2015) señalan que hay evidencia contundente para demostrar una "brecha de las noticias": las preferencias de periodistas no corresponden a las de lectores y lectoras de noticias. Mientras que los primeros promueven noticias sobre asuntos públicos, los segundos prefieren leer sobre asuntos no públicos. En su opinión, esta brecha informativa solo se revierte durante momentos importantes de actividad política, notablemente elecciones presidenciales. En esos casos, las personas sí buscan información sobre asuntos públicos como política, economía y noticias internacionales. En su opinión, esta tendencia tiene implicaciones importantes: quienes leen noticias tienden a comportarse como una "ciudadanía monitorial", término acuñado por Schudson (1998). Según Zaller (2003), esto contribuye a fomentar una "ciudadanía de alarma" que responde principalmente a mensajes en los medios de comunicación sobre "asuntos urgentes" de manera "emocionada y ruidosa" (p. 122).

Sin embargo, algunos críticos señalan que no hay evidencia suficiente para llegar a 
esa conclusión. En palabras de Meijer y Kormelink (2015), "cliquear o no cliquear [una noticia] no demuestra automáticamente la existencia de una brecha" entre la oferta de contenido sobre asuntos público y las preferencias por contenidos sobre asuntos no públicos (p. 676). De manera similar, Tenenboim y Cohen (2015) consideran que la preferencia por asuntos públicos no depende solamente de coyunturas electorales puesto que es posible encontrar patrones de lectura de noticias sobre política en momentos de vida política rutinaria o post electoral.

Esta investigación plantea revisitar los aportes de las ciencias sociales (específicamente la llamada "brecha de las noticias") desde los principios analíticos formulados por la ciencia de los datos o data science. En términos generales, este campo interdisciplinario, que incluye, entre otras, la estadística, el aprendizaje mecánico, la computación, el análisis de redes sociales y la comunicación, permite desarrollar metodologías, teorías, tecnologías y aplicaciones para el procesamiento científico de big data (Cao, 2016). Según Emani, Cullot y Nicolle (2015), se considera big data a aquellos conjuntos de datos con al menos las siguientes características:

$\diamond \quad$ Volumen: el tamaño del conjunto de datos supera la capacidad de sistemas de almacenamiento tradicionales, tales como bases de datos relacionales, para capturar, almacenar, gestionar y analizar su contenido.

$\diamond \quad$ Variedad: los datos no tienen una estructura única u homogénea, por el contrario, son una mezcla de diversos tipos, formatos y contenidos.

$\diamond \quad$ Velocidad: implica flujos acelerados y cambiantes de datos, no solamente para su generación, sino también para su procesamiento y análisis.

Así, la "brecha de las noticias" es un fenómeno adecuado para su estudio mediante data science, en particular considerando que los datos de interés provienen de grandes volúmenes producto de interacciones en redes sociales, no poseen una estructura única, contienen múltiples formatos y varían rápidamente en el tiempo.

\section{DISEÑO DE INVESTIGACIÓN: ANÁLISIS DE REDES SOCIALES Y ANÁLISIS DE CONTENIDO}

Esta investigación se sustenta en una metodología mixta que combinó la aplicación de visualizaciones y métricas asociadas al análisis de redes sociales (social network analysis) (Monge y Contractor, 2003; Wasserman y Faust, 1997) y el análisis de contenido (Krippendorff, 1990). Para evitar sesgos asociados al tipo de contenido que suele publicarse en medios de comunicación ciertos días de la semana (por ejemplo, abundancia de contenido deportivo los lunes), se optó por el método de la "semana construida" o "compuesta" (Hester y Dougall, 2007). En la práctica, este método se implementa al seleccionar el lunes de la primer semana, el martes de la segunda, el miércoles de la tercera y así sucesivamente hasta completar una "semana compuesta" con información de siete semanas distintas.

De este modo, se reconstruyeron dos semanas de informaciones publicadas en enero, febrero, julio y agosto de 2016, respectivamente. Específicamente, las fechas analizadas fueron: domingo 03 de enero, lunes 11 de enero, martes 19 de enero, miércoles 27 de enero, jueves 04 de febrero, viernes 12 de febrero, sábado 20 de febrero, domingo 03 de julio, lunes 11 de julio, martes 19 de julio, miércoles 27 de julio, jueves 04 de agosto, viernes 12 de agosto y sábado 20 de agosto, todos del año 2016.

Utilizando la aplicación informática Netvizz, se recolectó toda la información publicada en Facebook por seis medios de comunicación locales durante los días seleccionados: $\mathrm{CRHoy}$, Diario Extra, El Financiero, La Nación, Semanario Universidad y Tico Times. Para este estudio, se privilegiaron sitios de noticias que tuvieran o hubieran tenido una versión impresa, a excepción de CRHoy, que se seleccionó por ser el sitio de noticias con el mayor número de seguidores en Facebook.

Una vez recuperados los datos, se les aplicaron algoritmos de visualización y métricas para identificar patrones estructurales, utilizando el 
análisis de redes sociales. En esencia, esta metodología operacionaliza todo "patrón de interacción en un sistema dado [como] una red, las partes individuales de un sistema se denotan como nodos (nodes) y sus interacciones como lazos o aristas (edges)" (Oh y Monge, 2016, p. 1). En el caso de redes de comunicación, según Shumate y Contractor (2013), "las relaciones entre varios tipos de actores [...] ilustran las formas en que mensajes son transmitidos, intercambiados o interpretados" (p. 449). Para implementar este método, se analizaron los datos con la ayuda de software especializado para la visualización de datos.

De manera paralela, se constituyó una muestra de noticias para realizar un análisis de contenido. Para obtener esta muestra, se establecieron dos criterios: 1) la cantidad de interacciones registradas por cada noticia; 2) la frecuencia de publicación de los sitios de noticias. Es decir, se procuró que la muestra reflejara cuáles contenidos obtuvieron más interacciones de parte de usuarios, pero también que estuviera compuesta por noticias provenientes de todos los sitios informativos estudiados, no solo de los más populares. Específicamente, se escogió el primer y último quintil de noticias publicadas por los distintos perfiles informativos en cada uno de los días seleccionados. En otras palabras, se estudiaron las noticias más populares y menos populares de los seis sitios de noticias en conjunto. De este modo, se redujo el dominio de CRHoy en la muestra y se le dio más espacio a sitios como La Nación que, aunque no es el que recibe más interacciones, es uno de los que más publicaciones diarias realiza.

En total, la muestra estuvo constituida por 919 noticias que se codificaron según las siguientes variables: fecha, medio de comunicación, formato de la noticia, tema principal, si la noticia era sobre asuntos públicos o no, fuente de la noticia, sección donde fue compartida, cantidad de comentarios, cantidad de likes y cantidad de shares ${ }^{3}$. Los criterios

3 Dado que en enero de 2016 Facebook no había implementado las nuevas formas de expresar interacción con contenido en la plataforma (esto es, "like", "love", "haha", "wow", "sad" y "angry") no se reportan estos datos en este artículo. de codificación fueron definidos con base en estudios similares previos (Boczkowski y Mitchelstein, 2015; de Vreese, Esser y Hopmann, 2016) y se implementaron después de varias rondas de conversación y de codificación preliminar. Las dudas surgidas durante el proceso de codificación fueron resueltas de manera consensuada.

\section{¿QUÉ LIKEAN, COMENTAN Y COMPARTEN}

LAS PERSONAS USUARIAS DE SITIOS

COSTARRICENSES DE NOTICIAS?

A continuación, se analizan distintos resultados de esta investigación respecto de tres temas concretos:

1) Las estadísticas de publicación de sitios costarricenses de noticias en Facebook.

2) Las preferencias temáticas de usuarios expresadas por comportamientos como dar like, compartir y comentar contenidos noticiosos.

3) Los patrones que surgen al examinar los comportamientos de usuarias y usuarios como una red de interacciones entre noticias $y$ lectores.

1. ESTADÍSTICAS DE PUBLICACIÓN DE CONTENIDOS EN SITIOS DE NOTICIAS COSTARRICENSES EN FACEBOOK

En primera instancia, se contabilizaron las publicaciones de los sitios informativos seleccionados durante el periodo del estudio. De ese modo, fue posible identificar algunas dinámicas relevantes. La tabla 1 y el gráfico 1 resumen la información más significativa en ese sentido. En esta tabla, se reporta el número promedio de posts o publicaciones por cada sitio de noticias analizado, la cantidad de interacciones generadas por usuarios y el promedio de usuarios que interactuaron directamente con cada publicación. El gráfico 1 refleja el promedio de publicaciones diarias en Facebook efectuadas por los seis sitios de noticias de la muestra en la semana compuesta de julio y agosto de 2016. 
TABLA 1

PROMEDIO DE PUBLICACIONES, ENGAGEMENT Y USUARIOS POR PUBLICACIÓN 2016

\begin{tabular}{|c|c|c|c|c|c|c|c|c|c|c|c|c|}
\hline & \multicolumn{2}{|c|}{ CRHOY } & \multicolumn{2}{|c|}{ EXTRA } & \multicolumn{2}{|c|}{ FINANCIERO } & \multicolumn{2}{|c|}{ NACIÓN } & \multicolumn{2}{|c|}{ SEMANARIO } & \multicolumn{2}{|c|}{ TICO TIMES } \\
\hline & $S 1$ & $\$ 2$ & 51 & 52 & S1 & 52 & s1 & $\$ 2$ & s1 & $\$ 2$ & $\$ 1$ & $\$ 2$ \\
\hline POSTS & 37 & 50 & 46 & 37 & 21 & 17 & 53 & 53 & 2 & 4 & 7 & 3 \\
\hline ENGAGEMENT & 325,761 & 473,538 & 191,585 & 142,444 & 30,582 & 13,198 & 72,257 & 96,850 & 4601 & 5010 & 2804 & 2890 \\
\hline USUARIOS POR POST & 619 & 695 & 249 & 263 & 118 & 70 & 112 & 165 & 109 & 115 & 38 & 86 \\
\hline
\end{tabular}

Fuente: Elaboración propia.

GRÁFICO 1

CANTIDAD DE PUBLICACIONES DIARIAS

JULIO-AGOSTO, 2016

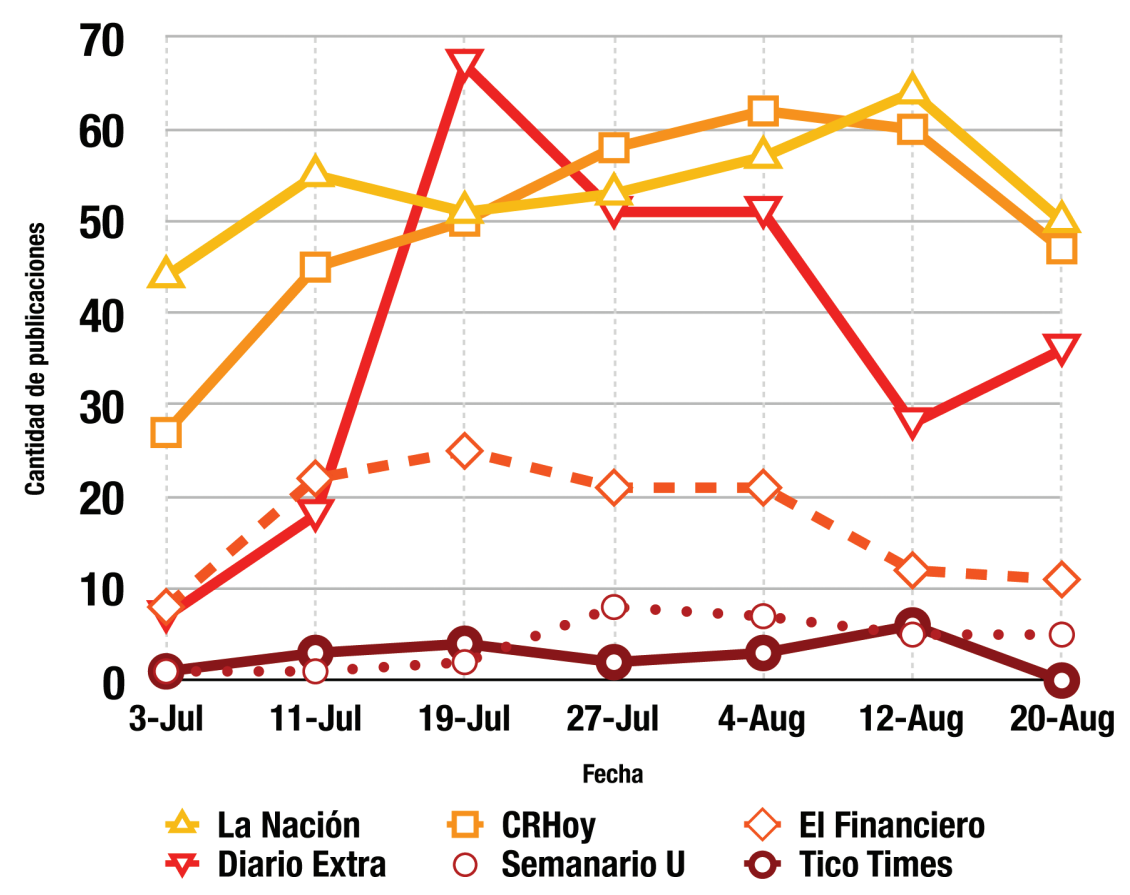

Fuente: Elaboración propia.

Se identificó una clara diferencia en la cantidad de usuarios que interactuaron con los contenidos de CRHoy.com respecto a los demás sitios de noticias. CRHoy.com obtuvo de manera regular un número de interacciones considerablemente más alto por cada post en Facebook. Mientras que otros sitios informativos pueden publicar hasta dos veces más que CRHoy.com en días regulares de actividad, CRHoy.com puede sextuplicar la cantidad de usuarios que interactuaron con sus contenidos a los de sus competidores 
más cercanos. A modo de ejemplo, durante la semana compuesta con información de enero y febrero de 2016, La Nación publicó un promedio de 53 veces por día en su perfil de Facebook y obtuvo un promedio de 5950 usuarios que interactuaron con sus contenidos, para un promedio de 112 personas que interactuaron por publicación. En el caso de CRHoy.com, se publicó un promedio de 37 contenidos por día durante la misma semana, pero un promedio de 22885 personas interactuaron con esos contenidos, es decir, casi 619 personas por post. El gráfico 2 refleja de manera clara esta diferencia a favor de CRHoy.com.

\section{GRÁFICO 2 \\ ENGAGEMENT DIARIO EN FACEBOOK \\ JULIO-AGOSTO, 2016}

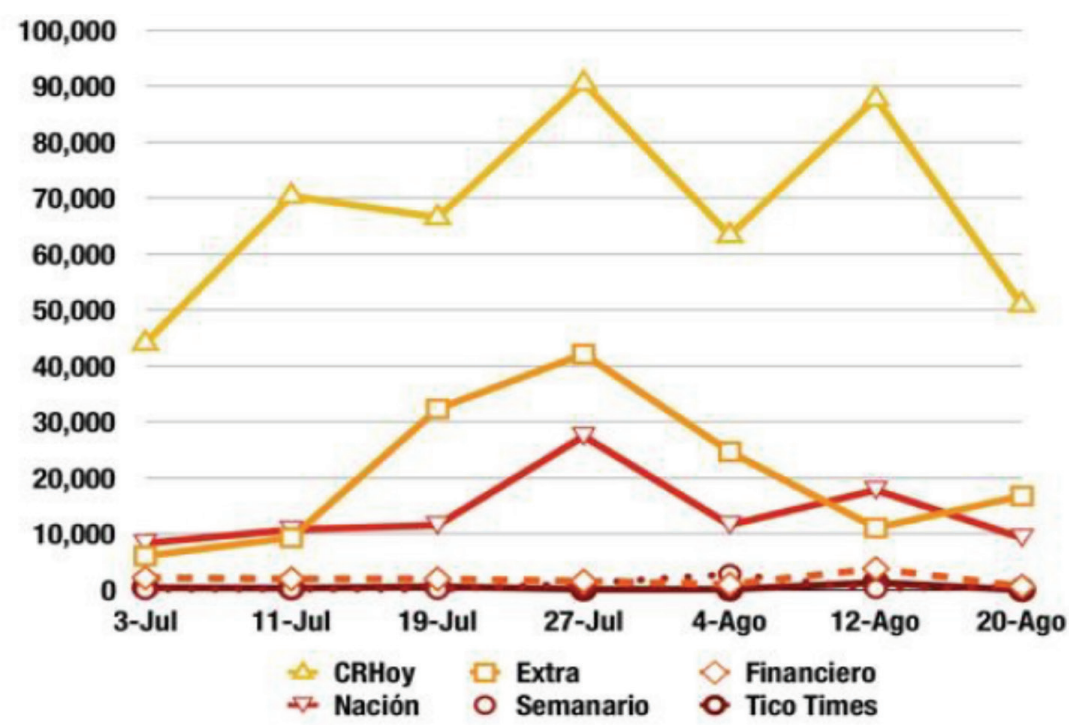

Fuente: Elaboración propia.

Una mirada a la cantidad de usuarios que interactuaron con los perfiles de estos sitios informativos también evidencia el claro dominio de CRHoy.com en la muestra. El 27 de julio de 2016, por ejemplo, se registraron un total de 90507 interacciones en el perfil de CRHoy. com en Facebook. Este representa el número más alto de interacciones con algún perfil noticioso durante el periodo en estudio. A modo de comparación, para alcanzar lo que CRHoy.com consiguió ese único día, Diario Extra tendría que duplicar y La Nación tendría que triplicar el número máximo de interacciones diarias registradas en su perfil.

Si se considera la semana compuesta con información de julio y agosto de 2016, se puede apreciar que publicar más información en Facebook sí resulta en un aumento del engagement con el perfil, pero no necesariamente mejora el número de usuarios que interactúan por post. Durante esa semana, por ejemplo, La Nación publicó la misma cantidad promedio de posts diarios (53) que la semana compuesta de enero $y$ febrero. Sin embargo, aumentó la cantidad de personas que interactuaron con su perfil $(8,723)$ y subió ligeramente el promedio de usuarios que interactúan por publicación (165). Por su parte, CRHoy.com aumentó de manera significativa el número de publicaciones diarias (50) y se incrementó notablemente el número total de personas que interactuaron con el contenido (34 766). Sin embargo, el incremento de 
usuarios que interactuaron (695) por post fue mucho más moderado que el de La Nación. Del mismo modo, Diario Extra incrementó ligeramente el número de usuarios que interactuaron por post a pesar de disminuir el número promedio de publicaciones diarias y el total de personas que interactuaron en su perfil.

\section{LO MÁS “LIKEADO", COMENTADO Y COMPARTIDO}

Al codificar los temas que recibieron más interacciones de parte de las usuarias y los usuarios de sitios costarricenses de noticias en Facebook, se comprobó la preferencia por ciertos temas durante ambas semanas compuestas (gráficos 3, 4 y 5).

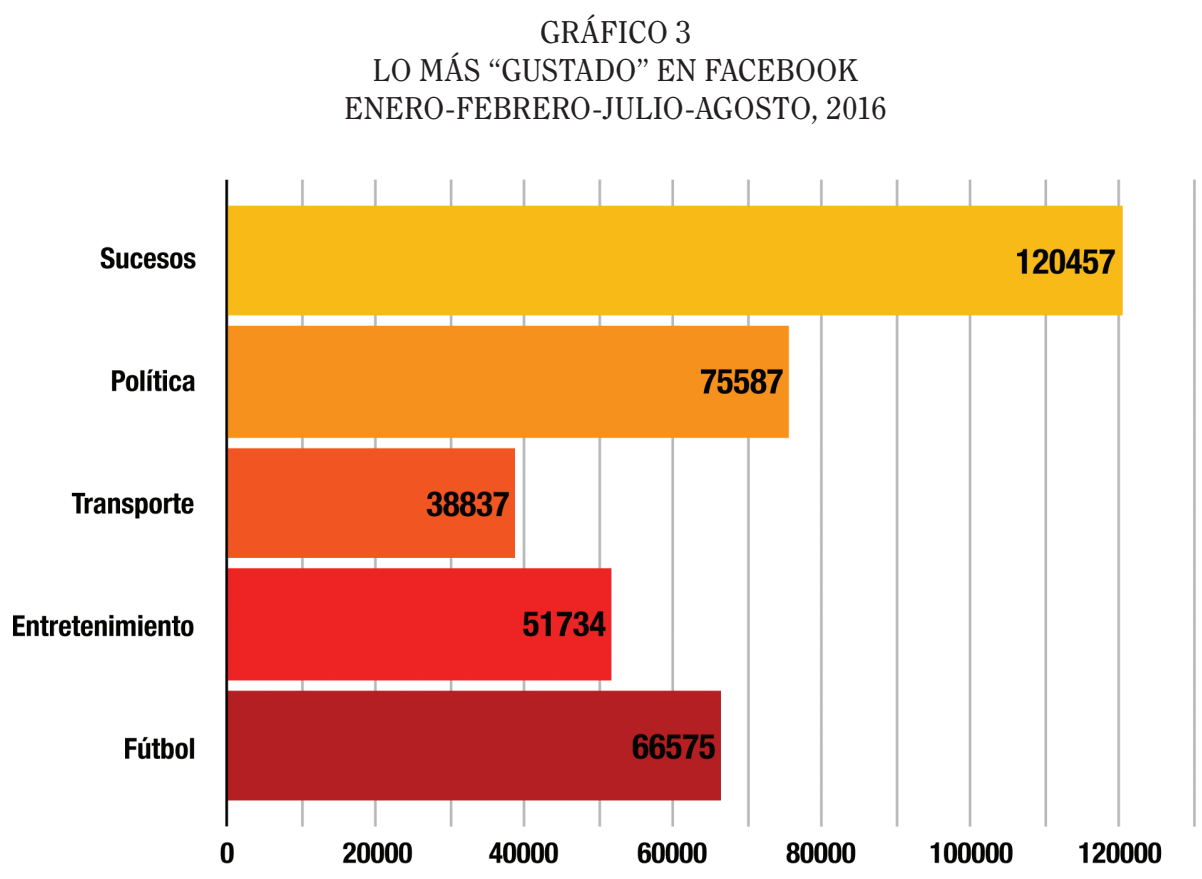

Fuente: Elaboración propia. 
GRÁFICO 4

LO MÁS COMENTADO EN FACEBOOK

ENERO-FEBRERO-JULIO-AGOSTO, 2016

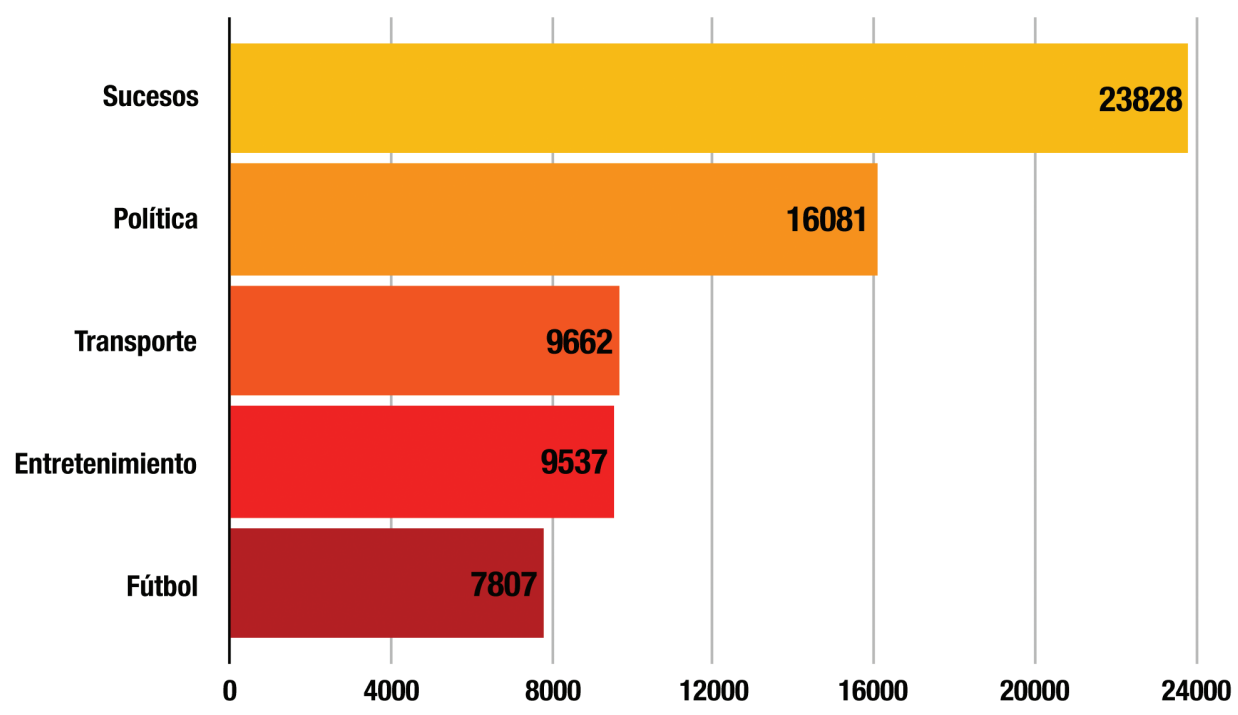

Fuente: $\quad$ Elaboración propia.

GRÁFICO 5

LO MÁS COMPARTIDO EN FACEBOOK

ENERO-FEBRERO-JULIO-AGOSTO, 2016

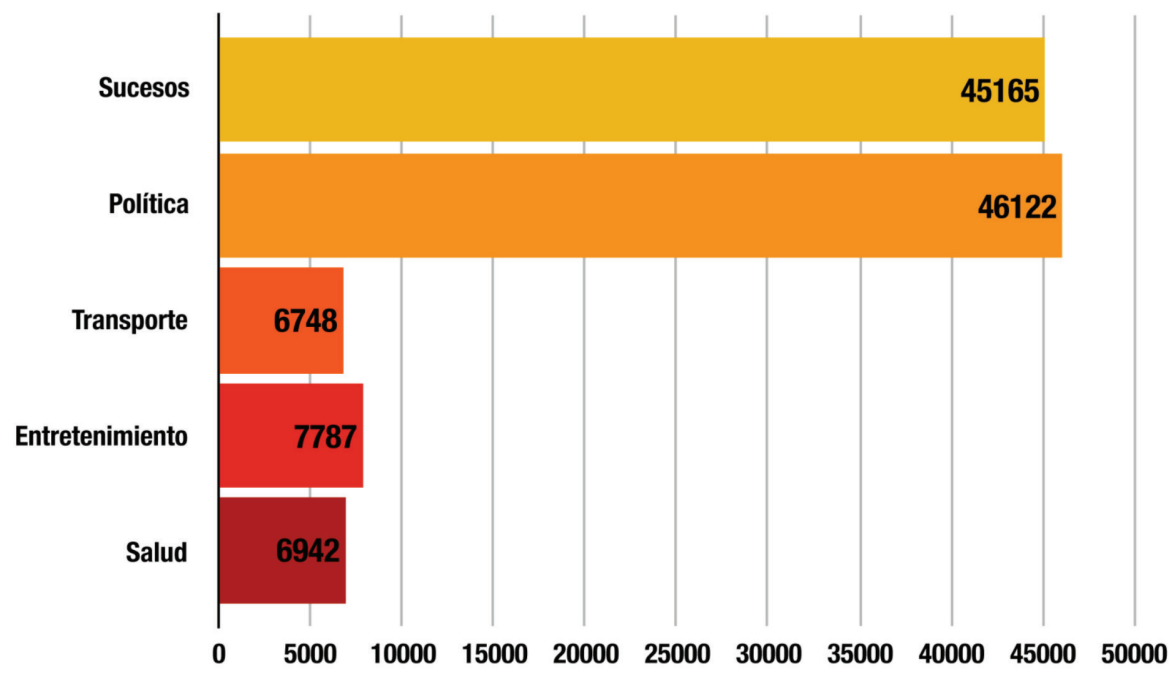

Fuente: Elaboración propia. 
Como puede apreciarse, los temas que recibieron más atención fueron sucesos y política, seguidos de fútbol, entretenimiento $y$ transporte. Considerando los datos de todos los medios de la muestra durante los cuatro meses estudiados, los sucesos fueron el tema con más "likes", el más comentado y el segundo más compartido. Llama la atención la presencia del tema de política (el cual combinó noticias sobre gobierno, funcionarios públicos, instituciones públicas, partidos políticos, presidencia, entre otros) entre los dos con mayor engagement. En general, podría afirmarse que los temas que recibieron mayor interacción por parte de usuarias y usuarios son las noticias sobre asuntos no públicos. Sin embargo, la presencia del tema de política sugiere que el interés por este tipo de noticias no debería despreciarse.

GRÁFICO 6

CONSUMO DE ASUNTOS PÚBLICOS Y ASUNTOS NO PÚBLICOS EN FACEBOOK

ENERO-FEBRERO-JULIO-AGOSTO, 2016

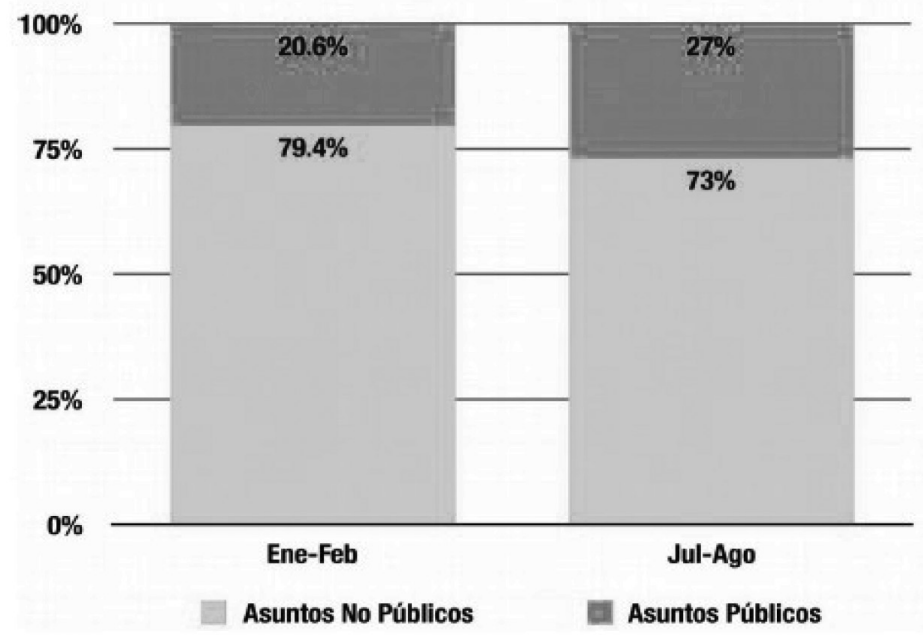

Fuente: Elaboración propia.

En la semana compuesta de enero y febrero, la preferencia por asuntos no públicos, considerando todos los sitios de noticias analizados, fue de 79,4\% frente a un 20,6\% dedicado a noticias de asuntos públicos. En el periodo correspondiente de julio y agosto, el 73\% de los contenidos más consultados en todos los sitios de noticias seleccionados tuvieron que ver con asuntos no públicos, en oposición al 27\% que podrían catalogarse como asuntos públicos. Inclusive, en El Financiero, en principio un medio dedicado a la discusión de asuntos eminentemente públicos como economía, la preferencia por asuntos públicos no es siempre clara: mientras que en enero y febrero de 2016, un $75,9 \%$ de las noticias con mayor interacción fueron sobre asuntos no públicos $(75,9 \%)$; en julio $y$ agosto del mismo año, este porcentaje se redujo a $43,5 \%$.

Esta preferencia tiende a acentuarse en los sitios de noticias que registran la mayor cantidad de interacciones: CRHoy, Diario Extra y La Nación. En pocas palabras, entre más consultado es un perfil de noticias en Facebook, más evidente es la preferencia por contenidos de asuntos no públicos. Por esa razón, es esperable que la preferencia de las lectoras y los lectores de medios de comunicación como CRHoy y Diario Extra, los dos sitios de noticias con el mayor número de interacciones de la muestra 
seleccionada, esté incidiendo en los promedios de toda la muestra. Por ejemplo, en la semana compuesta de julio y agosto de 2016, el $94,1 \%$ de las noticias con mayor interacción en CRHoy fueron sobre asuntos no públicos. Este es el porcentaje más alto de toda la muestra. Llama la atención que, en varias ocasiones, los porcentajes de noticias sobre asuntos no públicos con mayor número de interacciones publicadas por La Nación supera al de Diario Extra. En el periodo de enero y febrero, el 78,4\% de las noticias con más interacciones publicadas por La Nación en Facebook fue sobre asuntos no públicos, mientras que el equivalente en Diario Extra fue de 69,2\%.

En el caso de los sitios con menor interacción, hay un mayor balance en las preferencias por noticias sobre asuntos públicos $y$ las que no lo son. Si se considera al Semanario Universidad, el sitio informativo con la menor cantidad de posts en nuestra muestra, en la semana compuesta de julio y agosto de 2016, un $75 \%$ de las noticias que recibieron más interacción fueron sobre asuntos públicos.

\section{ANÁLISIS DE LAS REDES DE INTERACCIONES ENTRE USUARIOS Y NOTICIAS EN FACEBOOK}

Se identificó la existencia de patrones diferenciados en el comportamiento de las personas usuarias en cada uno de los medios analizados. Para detectar estos patrones, se utilizaron algoritmos de visualización y métricas de análisis de redes sociales (social network analysis). Los gráficos 7 y 8 reflejan estos comportamientos diferenciados en el consumo de noticias en los perfiles de Facebook de CRHoy y La Nación. En estos gráficos, cada nodo representa a una persona o noticia publicada por esos medios de comunicación en Facebook, y las aristas o enlaces entre los nodos representan las interacciones entre personas y noticias. Así, una mayor cantidad de nodos evidencia un mayor número de usuarios que interactuaron con las noticias, $y$ una mayor cantidad de aristas entre los nodos refleja un número mayor de personas que comentó, compartió o dio "me gusta" a esos contenidos noticiosos.

Con el propósito de detectar si había grupos o comunidades de personas interactuando con noticias específicas, se aplicó el algoritmo OpenOrd a los datos recolectados durante ambas semanas compuestas (Martin et ál., 2011). Este algoritmo agrupa los nodos en comunidades (también llamados módulos) de usuarias y usuarios cuyas interacciones se concentran en una noticia en particular. Cada comunidad de usuarios que interactuó con una noticia detectada por el algoritmo, recibió un color distinto en los gráficos 7 y 8 . 
GRÁFICO 7

INTERACCIONES ENTRE USUARIOS Y NOTICIAS DE CRHOY EN FACEBOOK

FEBRERO Y AGOSTO, 2016

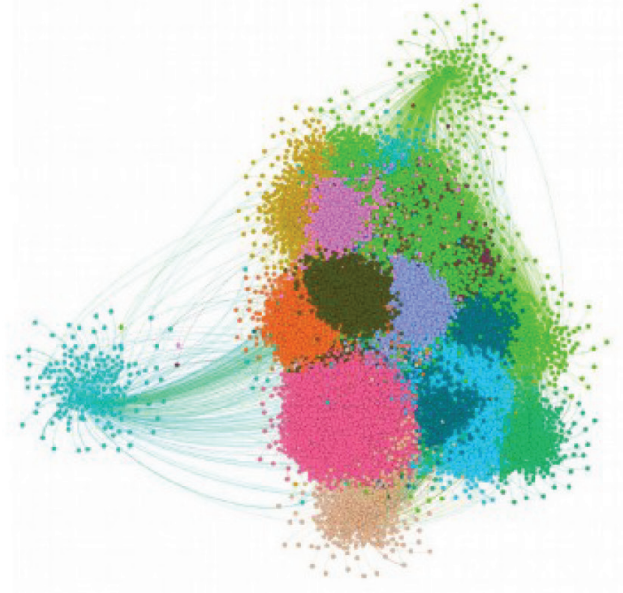

12-Feb-2016

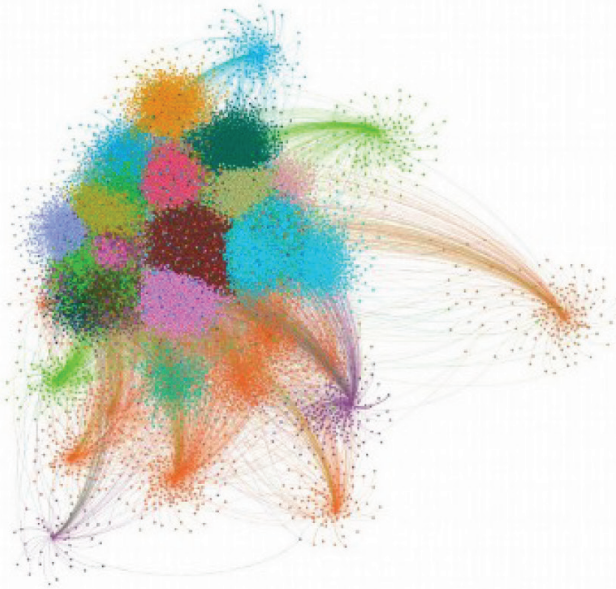

04-Ago-2016

Fuente: Elaboración propia.

GRÁFICO 8

INTERACCIONES ENTRE USUARIOS Y NOTICIAS DE LA NACIÓN EN FACEBOOK

FEBRERO Y AGOSTO, 2016

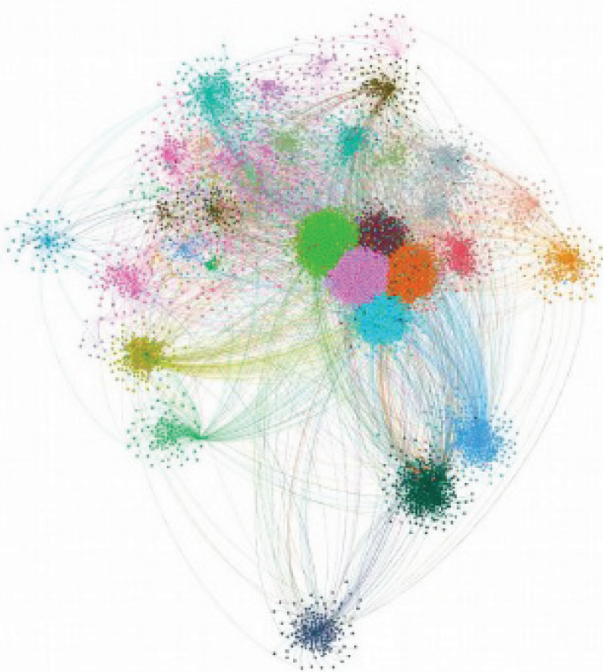

12-Feb-2016

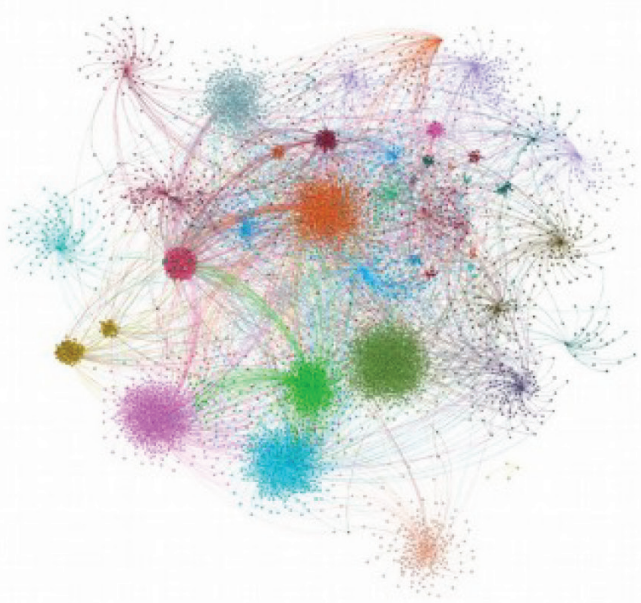

04-Ago-2016

Fuente: Elaboración propia.

(c) (1) (-) $\odot$ Rev. Ciencias Sociales Universidad de Costa Rica, 160: 37-55 / 2018 (II). (ISSN: 0482-5276) 
Al comparar los gráficos de cada sitio de noticias es posible constatar visualmente la existencia de patrones de comportamientos persistentes en el tiempo. En otras palabras, las usuarias y los usuarios tienden a reproducir estos comportamientos independientemente del momento del año o el día de la semana. Entonces, podría decirse que se trata de comportamientos estructurales que caracterizan la interacción de usuarios con las noticias de cada perfil en Facebook.

Otra constatación importante es que las personas no se comportan igual, ni interactúan del mismo modo, en cada sitio de noticias; es decir, las estructuras son específicas para cada medio. Estas diferencias se reflejan, por ejemplo, en los gráficos 7 y 8. La red de CRHoy es densa e interconectada, mientras que la de La Nación es desagregada y dispersa. En otras palabras, las personas que consultaron contenido en CRHoy interactuaron con más de una noticia (independientemente de si estas noticias estaban relacionadas temáticamente). En cambio, los lectores de La Nación interactuaron con una noticia, pero no necesariamente con otros contenidos publicados por el medio. Desde este punto de vista, la interacción con noticias en el perfil de Facebook de La Nación podría catalogarse como fragmentada.
Con el fin de comprobar cuantitativamente las diferencias estructurales evidentes en las visualizaciones obtenidas mediante OpenOrd, se aplicó para cada red de cada medio, en cada uno de los días estudiados, un conjunto de métricas clásicas de análisis de redes sociales, a saber: grado nodal, densidad y grado de modularidad (Wasserman y Faust, 1997). La métrica con mayor poder explicativo resultó ser el grado de modularidad, el cual se entiende como una medida que representa la facilidad con la que una red puede ser dividida en módulos (comunidades o grupos) relativamente independientes entre sí. El grado de modularidad varía de 0 a 1 , entre más cercano sea el valor a 1, mayor será la posibilidad de fragmentar la red en módulos (Caliandro y Gandini, 2017). De ese modo, se pudo confirmar que los gráficos 7 y 8 reflejan una diferencia estructural: en Facebook, el consumo de noticias en La Nación es significativamente más fragmentado que en CRHoy.

Los gráficos 9 y 10 muestran la modularidad de los medios analizados en las dos semanas compuestas recolectadas. Cabe aclarar que el Semanario Universidad fue descartado de los gráficos, ya que su modularidad fue prácticamente 0 en todo momento, dada la poca actividad del medio en Facebook durante el período de estudio. 
GRÁFICO 9

MODULARIDAD DE LOS MEDIOS DURANTE LA PRIMERA SEMANA COMPUESTA ENERO-FEBRERO, 2016

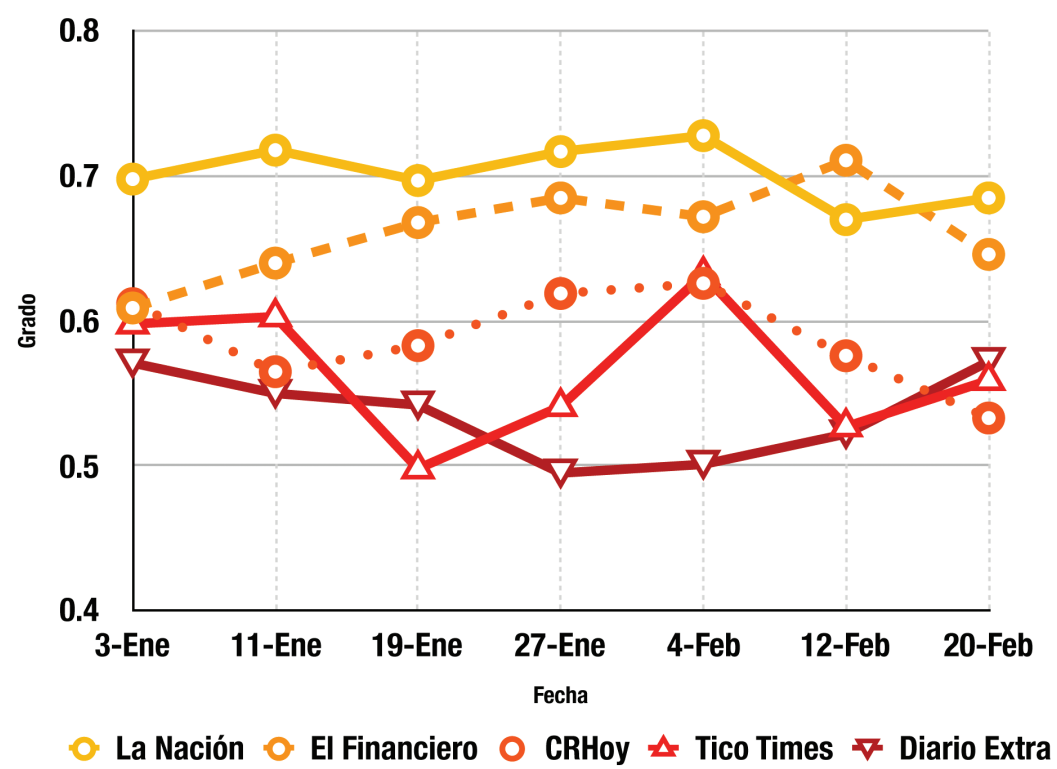

Fuente: Elaboración propia.

GRÁFICO 10

MODULARIDAD DE LOS MEDIOS DURANTE LA SEGUNDA SEMANA COMPUESTA JULIO-AGOSTO, 2016

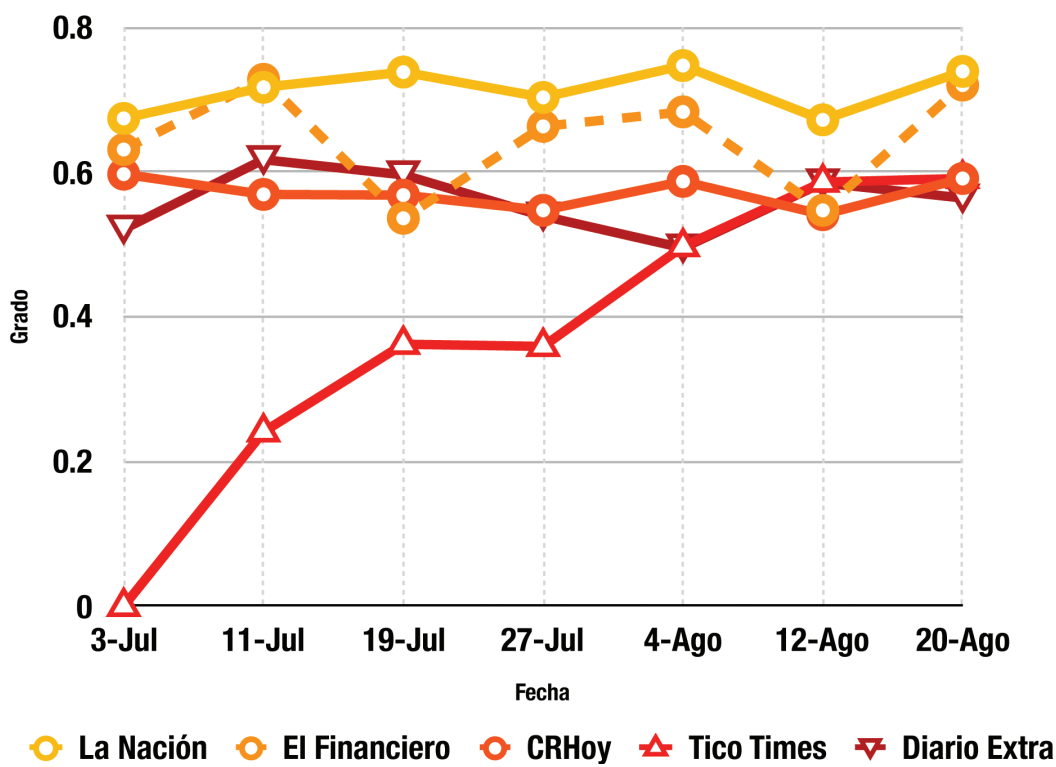

Fuente: Elaboración propia. 
Como puede observarse, con excepción del Tico Times, el comportamiento de la modularidad en ambas semanas estudiadas es muy similar. La Nación permanece como el medio con mayor modularidad durante todo el período de estudio, es decir, es la red más fácilmente separable en comunidades independientes enfocadas en noticias particulares (en otras palabras, el medio cuyo consumo de noticias es más fragmentado). Por su parte, CRHoy se mantiene considerablemente por debajo de $L a$ Nación, lo cual implica que su red es menos susceptible a ser fragmentada. Es importante insistir que las métricas de modularidad complementan las visualizaciones generadas en primera instancia por OpenOrd y contribuyen a validar los resultados obtenidos por este algoritmo. El patrón de comportamiento de la modularidad en cada medio puede considerarse como un identificador único (una especie de "huella") de su estructura y de las dinámicas de sus usuarias y usuarios.

\section{HACIA UNA MEJOR COMPRENSIÓN DEL} UNIVERSO INFORMATIVO DE FACEBOOK

En este artículo, se discutieron algunos patrones $y$ tendencias en el comportamiento de usuarias y usuarios de los perfiles de sitios costarricenses de noticias en Facebook. La investigación utilizó una metodología mixta (que combinó el análisis de contenido y el análisis de redes sociales) sobre un conjunto de datos considerables como big data, dada su heterogeneidad, variabilidad y volumen. Al indagar sobre las "huellas" digitales que dejan estas personas usuarias durante cuatro meses de 2016 en sus interacciones con contenido noticioso, esta investigación procuró suplementar estudios existentes que privilegian "autoinformes" de comportamientos, es decir; lo que los usuarios afirman haber leído. De esta forma, se buscó contribuir a una mejor comprensión de las dinámicas de circulación de contenido en una plataforma en claro crecimiento como fuente de noticias en el país.

\section{LIMITACIONES DEL ESTUDIO}

Antes de discutir algunas de las implicaciones de este estudio para investigaciones a futuro, resulta importante reconocer algunas de las limitaciones de este trabajo. En primer lugar, esta investigación está basada únicamente en seis sitios costarricenses de noticias. Dado que la muestra fue definida en función de criterios específicos (notablemente la vinculación original de los sitios de noticias con una versión impresa), varios de los perfiles de organizaciones noticiosas del país con más seguidores en Facebook fueron excluidos de la investigación. Es necesario reconocer que la inclusión de este conjunto adicional de actores, podría alterar algunos de los patrones identificados. Para resolver esta limitación, actualmente el equipo de investigación se encuentra analizando informaciones publicadas en redes sociales por un total de 10 medios de comunicación, incluyendo medios televisivos y radiofónicos, durante períodos más largos (de manera preliminar, es posible constatar que el estudio de esta muestra más completa de medios de comunicación confirma los resultados reportados en este artículo).

De manera similar, esta investigación se basó enteramente en la lectura de clics y registros digitales dejados por personas al interactuar con un contenido en Facebook. Sin embargo, la lectura de una noticia (en papel o en la pantalla) representa una práctica multidimensional que no debe equipararse o reducirse al clic (Bird, 2014). Por eso, para entender con mayor profundidad los resultados alcanzados en este estudio, se requiere analizar prácticas de lectura de noticias digitales desde aproximaciones cualitativas. Por ejemplo, métodos como las entrevistas en profundidad, la observación y el seguimiento de patrones de los ojos mientras se interactúa con la noticia (eye tracking) podrían ayudar a entender qué sigue después del clic, es decir, qué significa leer una noticia en la pantalla (de un dispositivo móvil o desde una computadora) y en qué sentido se pueda decir que se trata de un acto de lectura.

Una tercera limitación de este estudio es que no se reportan los datos de noticias con más clics, mayor número de comentarios o las más compartidas de todo el 2016, aunque está basado en una muestra representativa. Como complemento a este trabajo de investigación, sería interesante repetir el ejercicio analítico 
utilizando el conjunto de las noticias que obtuvieron la mayor interacción de usuarias y usuarios en todo el año, no solo una muestra compuesta por información de cuatro meses. Esto permitiría confirmar algunas de las tendencias identificadas en este artículo o quizá identificar otros patrones importantes. Asimismo, otra posibilidad para profundizar en los resultados obtenidos sería considerar los eventos que obtuvieron mayor interacción de parte de usuarias y usuarios en todo el año (inclusive comparar estos patrones con las publicaciones que recibieron menos interacción).

\section{NUEVAS LÍNEAS DE INVESTIGACIÓN}

Los resultados de esta investigación también apuntan a sugerentes líneas de investigación futura. Se dividen estas recomendaciones en dos grupos: 1) análisis de la oferta de contenido noticioso en Facebook y 2) análisis de las implicaciones de los patrones que se identificaron en el consumo de estas noticias.

Dentro del primer grupo, sobre la oferta de contenidos noticiosos, una investigación futura podría identificar qué tipos de noticias ofrecen los sitios costarricenses de noticias en Facebook. En su mayoría, este tipo de ejercicios analíticos se ha centrado en los sitios Web de estas organizaciones noticiosas (el artículo de Tristán y Álvarez en este volumen demuestra las oportunidades analíticas generadas por este tipo de investigación). Sin embargo, poco se sabe de la oferta noticiosa que brindan estos sitios de noticias en Facebook ¿Qué caracteriza el menú informativo al que tienen acceso las y los costarricenses en Facebook? Dada la difícil coyuntura económica que atraviesan algunos medios de comunicación a nivel local e internacional (Carazo, 2015; Siles y Boczkowski, 2012), resulta indispensable conocer si los sitios de noticias mantienen su compromiso de producir contenido relevante para la ciudadanía o si han traducido el interés de sus audiencias por asuntos no públicos (identificado en esta investigación) en una mayor oferta de este tipo de contenidos.

Otra investigación a futuro podría indagar en las diferencias en los tipos de estrategia implementadas por los sitios costarricenses de noticias para atraer a lectores a sus sitios. Los casos de CRHoy y La Nación son reveladores en este sentido. Ambos sitios de noticias emplean estrategias radicalmente distintas para acompañar el contenido que comparten en Facebook. Mientras que La Nación recurre a textos largos $y$ explicativos del contenido que se comparte (que a menudo requiere clics adicionales para poder leerse de manera completa), CRHoy opta por textos breves o incluso por compartir enlaces a las noticias sin ningún acompañamiento textual. En este sentido, sería importante conocer si los patrones encontrados en esta investigación responden en alguna medida al tipo de estrategia empleada por estos sitios de noticias. Este tipo de análisis tendría implicaciones importantes para la práctica profesional de la comunicación.

El segundo grupo de investigaciones futuras para profundizar en estos resultados se centra en dinámicas de apropiación o consumo de contenido noticioso en Facebook. Por ejemplo, se hace urgente conocer las implicaciones políticas de los patrones que se identificaron en esta investigación. Otros autores han advertido sobre la posibilidad de que el consumo de noticias en Internet pueda estar asociado a una pérdida de interés en noticias cuyas posiciones ideológicas o temáticas difieren del punto de vista de quien las lee. Este tema se volvió una clave para entender el rol de los sitios de noticias en la elección presidencial de 2016 en Estados Unidos (Lakoff, 2016). En este sentido, una contribución investigativa importante radica en esclarecer si hay evidencia empírica para sostener esta preocupación.

El caso mencionado de la elección presidencial en Estados Unidos también ofrece pistas de posibles trabajos para profundizar en los resultados encontrados en esta investigación. Como se discutió en párrafos anteriores, autores como Boczkowski y Mitchelstein (2015) sugieren que la preferencia por noticias sobre asuntos no públicos tiende a revertirse durante eventos políticos como elecciones presidenciales. En el caso de Costa Rica, una investigación longitudinal haría posible entender si efectivamente cambian las preferencias de consumo informativo durante eventos especiales o si, 
por el contrario, tienden a mantenerse. Según autores como Schudson (1998), este tipo de datos permitiría una mejor comprensión de cuál es la capacidad que tiene la ciudadanía de ejercer su capacidad de control y monitoreo del Estado. De manera más general, autores como Williams y Delli Carpini (2011) han advertido sobre la artificialidad de la distinción entre asuntos públicos y no públicos. Siguiendo esta recomendación, sería importante indagar en el tipo de interacciones que caracterizan las conversaciones sobre ambos tipos de noticias, con el propósito de concluir si la distinción sigue siendo relevante para entender implicaciones políticas de la circulación de noticias.

Un tema central en estudios en comunicación es la influencia ejercida por los llamados "líderes de opinión" en las audiencias de medios de comunicación (Katz, 1957; Katz y Lazarsfeld, 1955). Esta línea de investigación sostiene que la influencia de los medios de comunicación nunca es directa sobre sus públi$\cos$. Al contrario, afirman estos autores, la figura de los "líderes de opinión" es determinante en la medida en que "filtran" los mensajes para otras personas y condicionan la forma en que estos mensajes se interpretan. Este estudio abre nuevas líneas de investigación para actualizar el análisis de líderes de opinión y dinámicas de influencia contemporáneas. La identificación de patrones en la circulación de noticias en Facebook invita a considerar qué tipo de figuras y cuáles personas tienen la capacidad de incidir en las formas en que ciertos contenidos y temas tienden a "viralizarse" en redes sociales.

Finalmente, otro tema importante que puede examinarse a la luz de los resultados de esta investigación tiene que ver con el uso que hacen las audiencias para conversar con otros sobre las noticias. Como Bird (2014) argumenta, para muchas personas, "el contenido de las noticias en sí mismas es menos importante que las varias actividades $y$ conexiones sociales que vienen con la atención a las noticias" (p. 494). Típicamente, este fenómeno ha sido estudiado mediante métodos cualitativos de investigación. Sin embargo, el auge de redes sociales y de las secciones de comentarios en los perfiles de los sitios de noticias en Facebook sugiere que algunas de estas dinámicas podrían estar migrando a espacios digitales y estar viviendo sugerentes transformaciones. En este sentido, las secciones de comentarios en los sitios de noticias requieren mayor atención analítica como un lugar de intercambio donde se lleva a cabo una negociación importante del significado de las noticias en la vida cotidiana de sus lectoras y lectores (el artículo de Kotras incluido en este volumen es un avance significativo en esa dirección).

El estudio de la circulación de noticias en línea es un ejemplo de lo que Law (2002) llama un objeto de investigación confuso o enmarañado (messy). Como Law argumenta, este tipo de fenómenos requiere de "montajes metodológicos" (method assemblages) que den cuenta de la complejidad y diversidad de dinámicas que los atraviesa y los compone. Dar "me gusta", comentar o compartir una noticia en Facebook es una práctica tanto comunicativa como política $y$ tecnológica. Al combinar los aportes de las ciencias sociales y el data science, con este artículo se espera haber contribuido parcialmente a la construcción de este tipo de abordajes teóricos y metodológicos. Siguiendo el argumento de Law, este tipo de aportes mejoraría parcialmente la comprensión de realidades complejas como la democracia contemporánea.

\section{REFERENCIAS}

Araya, J. (25 mayo de 2016). Internet y redes sociales avanzan, otros medios retroceden. Semanario Universidad. Recuperado de http://semanariouniversidad.ucr.cr/ pais/internet-redes-sociales-avanzanotros-medios- retroceden/

Bird, S. E. (2014). Seeking the audience for news: Response, news talk, and everyday practices. En V. Nightingale (Ed.), The handbook of media audiences (pp. 489508). Malden, ма: Wiley-Blackwell.

Boczkowski, P. J., y Mitchelstein, E. (2015). La brecha de las noticias: La divergencia entre las preferencias informativas de los medios y el público. Buenos Aires: Manantial.

Caliandro, A., y Gandini, A. (2017). Qualitative research in digital environments: $A$ 
research toolkit. New York: Taylor \& Francis.

Cao, L. (2016). Data science and analytics: A new era. International Journal of Data Science and Analytics, 1(1), 1-2.

Carazo, C. (2015). Periodistas y salas de redacción en Costa Rica frente a los retos del siglo ххг. San José: Editorial de la Universidad de Costa Rica.

de Vreese, C., Esser, F., y Hopmann, D. N. (Eds.) (2016). Comparing political journalism. London: Routledge.

El Financiero/unimer. (2015). Red 506. San José: El Financiero.

Emani, C. K., Cullot, N., y Nicolle, C. (2015). Understandable big data: A survey. Computer Science Review, 17, 70-81.

Habermas, J. (1989). The structural transformation of the public sphere: An inquiry into a category of bourgeois society. Cambridge: Polity Press.

Hester, J. B., y Dougall, E. (2007). The efficiency of constructed week sampling for content analysis of online news. Journalism \& Mass Communication Quarterly, 84 (4), 811-824.

Katz, E. (1957). The two-step flow of communication: An up-to-date report on an hypothesis. Public Opinion Quarterly, 21(1), 61-78.

Krippendorff, K. (1990). Metodología de análisis de contenido: Teoría y práctica. Barcelona: Paidós.

Lakoff, G. (15 de diciembre de 2016). How to help Trump. Georgelakoff.com. Recuperado de https://georgelakoff. com/2016/12/15/how-to-help-trump/

Law, J. (2004). After method: Mess in social science research. London: Routledge.

Madianou, M. (2010). Living with news: Ethnography and news consumption. En S. Allan (Ed.), The Routledge companion to news and journalism studies (pp. 428438). London: Routledge.

Martin, S., Brown, W. M., Klavans, R., y Boyack, K. W. (2011). OpenOrd: an opensource toolbox for large graph layout.
In Proceedings of SPIE (Vol. 7868). International Society for Optics and Photonics. Recuperado de https://doi. org/10.1117/12.871402

Meijer, I. C., y Kormelink, T. G. (2015). Checking, sharing, clicking and linking. Digital Journalism, 3(5), 664-679.

Monge, P. R., y Contractor, N. S. (2003). Theories of communication networks. Oxford: Oxford University Press.

Oh, P., y Monge, P. (2016). Network theory and models. En K. B. Jensen, R. T. Craig, J. D. Pooley y E. Rothenbuhler (Eds.), International encyclopedia of communication theory and philosophy (pp. 1-15). Hoboken, ss: Wiley-Blackwell.

Pew Research Center. (24 de febrero de 2010). Millennials: Confident. Connected. Open to Change. Pewsocialtrends. Recuperado de http://www.pewsocialtrends. org/2010/02/24/millennials-confidentconnected-open-to-change/

Pew Research Center. (1 de junio de 2015). Facebook top source for political news among millennials. Journalism.org. Recuperado de http://www.journalism. org/2015/06/01/facebook-top-source-forpolitical-news-among- millennials/

Schudson, M. (1998). The good citizen. New York: Free Press.

Shumate, M., y Contractor, N. (2013). The emergence of multidimensional social networks. En L. L. Putnam y D. K. Mumby (Eds.), The SAGE handbook of organizational communication (pp. 449474). Thousand Oaks, ca: Sage.

Siles, I., y Boczkowski, P. J. (2012). Making sense of the newspaper crisis: A critical assessment of existing research and an agenda for future work. New Media \& Society, 14(8), 1375-1394.

Tenenboim, O., y Cohen, A. A. (2015). What prompts users to click and comment: A longitudinal study of online news. Journalism, 16(2), 198-217.

Wasserman, S., y Faust, K. (1997). Social network analysis: Methods and applications. Cambridge: Cambridge University Press. 
Wellman, B. (1997). Análisis estructural de redes sociales: Del método y la metáfora a la teoría y la sustancia. Debates en Sociología, 22, 47-97.

Williams, B. A., y Delli Carpini, M. X. (2011). After broadcast news: Media regimes, democracy, and the new information environment. Cambridge: Cambridge University Press.
Zaller, J. (2003). A new standard of news quality: Burglar alarms for the monitorial citizen. Political Communication, 20, 109-130.

Fecha de ingreso: $24 / 10 / 2017$

Fecha de aprobación: 23/04/2018 
